\title{
Pre-treatment with spermine for acute cerebral ischemia/reperfusion injuries
}

\author{
HAI DU, XING MING and SHENGKUI ZHOU \\ Department of Neurosurgery, Xuzhou Central Hospital, Xuzhou, Jiangsu 221009, P.R. China
}

Received August 22, 2016; Accepted March 30, 2017

DOI: $10.3892 /$ etm.2017.4455

\begin{abstract}
Acute cerebral ischemia/reperfusion injury (ACIR) rat model was used to investigate the effect of spermine on oxidative stress and apoptosis. Sixty healthy and clean male Sprague-Dawley rats were randomly divided into 3 groups: The sham-operated group $(n=12)$, the model group $(n=12)$ and the experiment group $(n=36)$. The experiment group was further divided into 3 subgroups: The SPE-10 group ( $n=12)$, the SPE-25 group $(n=12)$ and the SPE-50 group $(n=12)$. Rats in the experimental sub-groups SPE-10, SPE-25 and SPE-50 were injected with 10,25 and $50 \mathrm{mg} / \mathrm{kg}$ of spermine, respectively, one week before the establishment of rat models. Rats in the sham-operated and model groups were injected with $0.9 \%$ $\mathrm{NaCl}$ solution. We evaluated the effect of spermine on malondialdehyde (MDA) level and superoxide dismutase (SOD) activity using ELISA kits. Bax and Bcl-2 levels were measured using western blot analysis. Our results showed that after spermine injection, MDA levels markedly decreased, while SOD activity increased significantly. The variations in MDA levels and SOD activity were dose (spermine)-dependent. Bax protein levels increased significantly, while Bcl-2 levels decreased significantly after the onset of ACIR injuries. After spermine injection, there was a significant decrease in Bax levels. Bcl-2 levels in these rats markedly increased. The observed decline in Bax levels and the increase in Bcl-2 levels in the experimental groups were dose-dependent. We concluded that spermine protected nerve tissues in rats with ACIR by decreasing the MDA level, increasing SOD activity and modifying the balance between $\mathrm{Bax}$ and $\mathrm{Bcl}-2$ proteins.
\end{abstract}

\section{Introduction}

Acute cerebral ischemia/reperfusion injury (ACIR) is a major disease that threatens health of the patients by causing excessive

Correspondence to: Dr Shengkui Zhou, Department of Neurosurgery, Xuzhou Central Hospital, 199 Jiefang Road, Xuzhou, Jiangsu 221009, P.R. China

E-mail: shengkuizhou9611@163.com

Key words: spermine, ischemia/reperfusion injury, oxidative stress, cell apoptosis release of oxygen-free radicals and inducing oxidative stress and inflammatory response to aggravate the neurological injuries $(1,2)$. Besides neurological injuries, oxygen-free radicals affect vascular endothelium and delay the repair of neurological tissues (3).

Spermine is a polyamine with the ability to bind to DNA, RNA and proteinase molecules and regulate cell proliferation, differentiation and apoptosis. Spermine is believed to be an indispensable factor in cellular metabolism (4). In this study, using on ACIR animal model, we investigated the effect of spermine on oxidative stress and apoptosis and obtained excellent results.

\section{Materials and methods}

Experimental animals. Sixty healthy and clean male Sprague-Dawley rats (provided by the Experimental Animal Center in Medical School of Xiamen University) with a gestational age ranging from 6 to 8 weeks and weight ranging from 250 to $270 \mathrm{~g}$ were used in this study. Before starting the experiments, the rats were kept and fed for one week in order for the animals to become acclimatized to their new environment. Rats were housed in a temperature controlled room $\left(21 \pm 2^{\circ} \mathrm{C}\right)$ on a $12: 12$-h light/dark cycle (lights on at 6:00 a.m.). All rats had free access to water and food. The rats were randomly divided into 3 groups: The sham-operated group $(n=12)$, the model group $(n=12)$ and the experiment group $(n=36)$. The experiment group was further divided into 3 subgroups: The SPE-10 group $(n=12)$, the SPE-25 group $(n=12)$ and the SPE-50 group $(n=12)$. Rats in the experimental sub-groups SPE-10, SPE-25 and SPE-50 were injected with 10,25 and $50 \mathrm{mg} / \mathrm{kg}$ of spermine, respectively, one week before the establishment of rat models. Rats in the sham-operated and model groups were injected with $0.9 \% \mathrm{NaCl}$ solution. Ethics approval was obtained from the Animal Ethics Committee of Xuzhou Medical University.

Reagents. We used Spermine (Sigma-Aldrich, St. Louis, MO, USA); malondialdehyde (MDA) and superoxide dismutase (SOD) ELISA kits for rats (Shanghai Gefan Biotechnology Co., Ltd., Shanghai, China); $1 \%$ pentobarbital, $10 \%$ paraformaldehyde, $0.9 \% \mathrm{NaCl}$ solution (Tianjin Chemreagent Co., Ltd., Tianjin, China), rat anti-Bax and anti-Bcl-2 monoclonal antibody, DAB coloration kits (Santa Cruz Biotechnology, Inc., Santa Cruz, CA, USA). 
Table I. Neurological function scale after onset of ACIR injuries in each group.

\begin{tabular}{lcccc}
\hline Group & $\mathrm{n}$ & $24 \mathrm{~h}$ & $48 \mathrm{~h}$ & $72 \mathrm{~h}$ \\
\hline Sham-operated group & 12 & 0 & 0 & 0 \\
Model group & 12 & $3.71 \pm 0.32^{\mathrm{a}}$ & $2.84 \pm 0.30^{\mathrm{a}}$ & $2.33 \pm 0.24^{\mathrm{a}}$ \\
SPE $(10 \mathrm{mg} / \mathrm{kg})$ & 12 & $3.14 \pm 0.35^{\mathrm{b}}$ & $2.42 \pm 0.28^{\mathrm{b}}$ & $1.87 \pm 0.21^{\mathrm{b}}$ \\
SPE $(25 \mathrm{mg} / \mathrm{kg})$ & 12 & $2.85 \pm 0.31^{\mathrm{b}}$ & $2.31 \pm 0.26^{\mathrm{b}}$ & $1.74 \pm 0.22^{\mathrm{b}}$ \\
SPE $(50 \mathrm{mg} / \mathrm{kg})$ & 12 & $2.77 \pm 0.29^{\mathrm{b}}$ & $2.10 \pm 0.23^{\mathrm{b}}$ & $1.52 \pm 0.19^{\mathrm{b}}$ \\
\hline
\end{tabular}

${ }^{\mathrm{a}} \mathrm{P}<0.05$, compared with the sham-operated group; ${ }^{\mathrm{b}} \mathrm{P}<0.05$, compared with the model group. ACIR, acute cerebral ischemia/reperfusion injury.

Establishment of ACIR animal models. Rats received an intraperitoneal injection of $1 \%$ pentobarbital for anesthesia, and were fixed on the operating table. The common carotid artery, internal and external carotid arteries were isolated by a midline linear skin incision in the neck. External carotid artery and the end part of the common carotid artery proximal to the heart were all ligatured and the internal carotid artery was occluded by carotid clips. After the removal of carotid clips from internal carotid artery, the common carotid artery was sutured. Suture thread was advanced slowly $(\sim 2.0 \mathrm{~cm})$ towards the head, and suture thread and the ligatured internal carotid artery were fixed and seamed layer by layer. After $2 \mathrm{~h}$, the suture thread was removed to establish the reperfusion model. In the sham-operated group we only isolated the relevant vessels.

Neurological function scale. Neurological function scale assessment was performed at 24, 48 and $72 \mathrm{~h}$ after establishment of the rat models according to the Longa 5-point scale of $0-4$. In this scale, 0 point was given for normal condition without any symptoms of neurological injury; 1 point, for cases incapable of extending upper limbs; those cases incapable of accomplishing contralateral forelimb internal rotation received 2 points; cases incapable of accomplishing contralateral bending motion received 3 points and those who are in coma or unconscious received 4 points.

Effect of spermine on MDA level and SOD activity. Seventy-two hours after establishment of the models, the spinal cords of the rats were extracted and MDA level as well as SOD activity were measured using ELISA kits. We closely followed the instructions provided by the kit manufacturer to realize these measurements. We used a microplate reader with the wavelength set as $450 \mathrm{~nm}$.

Bax and Bcl-2 protein expression levels. Seventy-two hours after establishment of the models, brain tissue was extracted. Tissue was ground and treated with $3 \mathrm{ml} / \mathrm{g}$ of protein lysis buffer. Samples were then centrifuged at $1 \times 10^{4} \mathrm{~g}$ for $40 \mathrm{~min}$. Total protein concentration was measured using BCA assay. For western blot analysis, protein samples were subjected to SDS-PAGE followed by transfer to a membrane. Each membrane was blocked and incubated with rabbit monoclonal Bax antibody (dilution, 1:500; cat. no. ab32503) and rabbit monoclonal Bcl-2 antibody (dilution, 1:500; cat. no. ab32124) (Abcam, Cambridge, MA, USA) overnight. After rinsing, the membrane was incubated with secondary goat anti-rabbit (HRP) IgG antibody (dilution, 1:2,000; cat. no. ab6721; Abcam) for $3 \mathrm{~h}$. Finally, color developer was added and results were compared with reference GAPDH. We used a GEL imaging system and the gray value was calculated using ImageJ software (version X; Media Cybernetics, Silver Springs, MD, USA). The relative gray value was calculated as: (assayed gray value of protein/internal reference gray value of GAPDH) x $100 \%$.

Statistical analysis. SPSS 20.0 (IBM, Armonk, NY, USA) was used for our statistical analysis. Measurement data are presented as mean \pm standard deviation. For intergroup comparisons we used single factor ANOVA analysis. LSD test was used for paired comparisons and variance analysis of repeated measurement was performed for intragroup comparisons. $\mathrm{P}<0.05$ was considered to indicate a statistically significant difference.

\section{Results}

Significant increases in neurological function scores were detected in different groups after the onset of ACIR injuries and when compared with the sham-operated group, differences were statistically significant $(\mathrm{P}<0.05)$. Neurological function scores in all the rats decreased over time; however rats in the experimental groups experienced a more significant decrease in these scores. Our results revealed that higher doses of spermine intensified the score reduction. Differences were statistically significant $(\mathrm{P}<0.05)$ (Table I).

After the onset of ACIR injuries, we detected a significant increase in MDA levels in all the rats. At the same time, a significant decrease in SOD activity was observed in all the rats $(\mathrm{P}<0.05)$. After spermine injection, MDA levels dcereased significantly while SOD activity increased significantly. Differences between the experimental groups and model group were statistically significant $(\mathrm{P}<0.05)$. In rats injected with different doses of spermine, the variations in MDA levels and SOD activity were dose-dependent $(\mathrm{P}<0.05)$ (Fig. 1).

Bax protein levels increased significantly, while Bcl-2 levels decreased significantly after the onset of ACIR injuries $(\mathrm{P}<0.05)$. After spermine injection, there was a significant drop in Bax levels, while the Bcl-2 levels increased obviously in these rats. Differences between the experimental groups and model group were statistically significant $(\mathrm{P}<0.05)$. In rats injected with different doses of spermine, the observed 

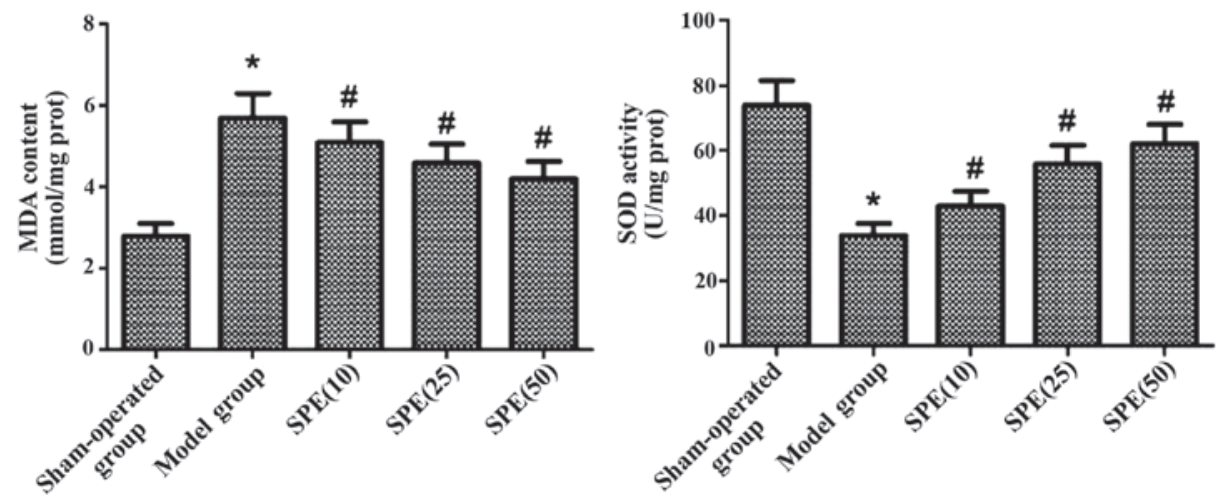

Figure 1. Effect of spermine on MDA content and SOD activity of rats $\left(\right.$ " $\mathrm{P}<0.05$, compared with the sham-operated group; ${ }^{\text {\# }} \mathrm{P}<0.05$, compared with the model group). MDA, malondialdehyde; SOD, superoxide dismutase.

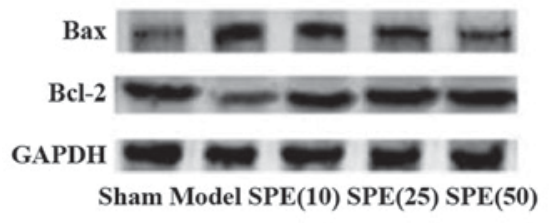

Sham Model SPE(10) SPE(25) SPE(50)
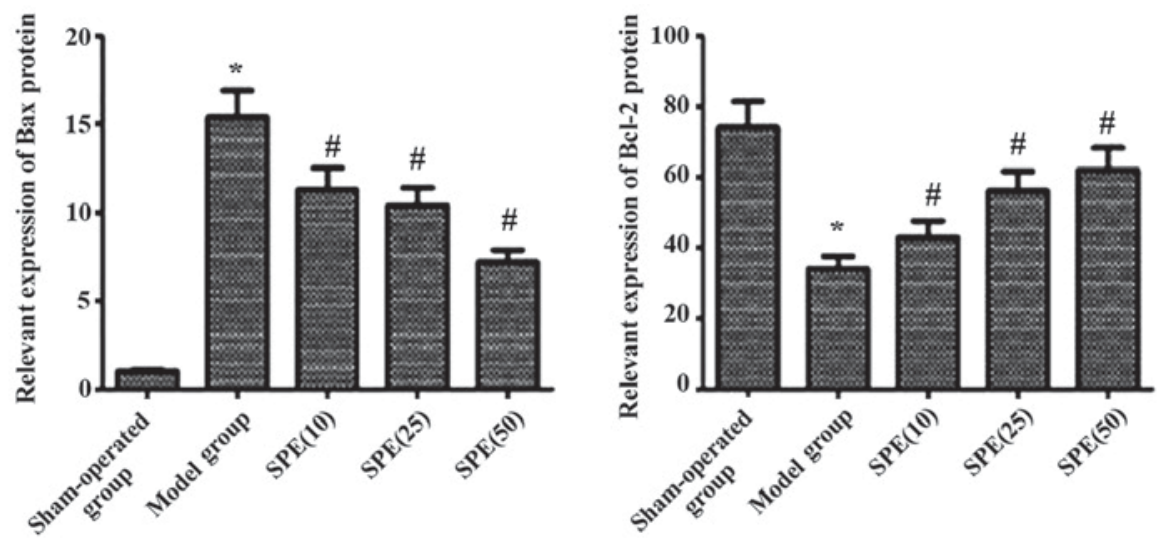

Figure 2. Effect of spermine on the expression of Bax and Bcl-2 protein in ACIR ("P $<0.05$, compared with the sham-operated group; "P<0.05, compared with the model group). ACIR, acute cerebral ischemia/reperfusion injury.

decrease in Bax levels and the increase in Bcl-2 were also dose-dependent $(\mathrm{P}<0.05)$ (Fig. 2).

\section{Discussion}

Pathological and physiological mechanisms involved in acute cerebral ischemia/reperfusion injuries are quite complex. Results obtained from previous studies suggested that a shortage in oxygen supply caused by acute cerebral ischemia, increased apoptosis as well as necrosis rate in neurons (5). This condition could trigger the release of substances such as proteolytic enzymes and reactive oxygen species (ROS). These substances could trigger the focal oxidative stress response. It has been shown that after the onset of ischemia, oxidative stress response aggravates neuron injuries (6). This is deemed to be a major mechanism for injuries secondary to the ACIR (6). Activated ROS facilitates the oxidative stress response induced by ACIR and further increases the expression of neuron-related apoptosis proteins in cerebral tissues (7). Prior studies showed that spermine played an important role in the pathological and physiological processes in tumors, diabetes and Alzheimer's disease (8). Amyloid $\beta$-peptide, usually found in brain tissues of Alzheimer patients, can activate the ROS in neurons and induce an oxidative stress response. However, spermine suppresses the generation of amyloid $\beta$-peptide and protects nerves and exerts an antioxidant effect (9). Additional findings suggested that spermine level in the ischemic region of brain was significantly low, therefore cells in this region were more susceptible to oxidative stress (10). Spermine can also increase the stability of chromosomes by eradicating the intracellular free radicals (11). Oxidative stress response is an important pathogenesis of ACIR. MDA level can directly reflect the level of internal oxygen radicals and indirectly reflect the injuries caused by these free radicals (12). SOD can protect cells from injuries caused by oxygen-free radicals and SOD level can indicate the activity of the oxygen-free radical endogenous scavenging system (13).

In this study, we showed that spermine improved the neuron condition by inhibiting the oxidative stress response and alleviated injuries caused by cerebral ischemia-reperfusion. Bcl-2 protein family has been proven to be involved in apoptosis (14). $\mathrm{Bcl}-2$ is an important anti-apoptosis protein in Bcl family that can protect neurons and inhibit cell proliferation. Bcl-2 also suppresses cytochrome $c$ (Cyt $\mathrm{C}$ ) and caspase by acting on the mitochondrial membrane (15). As a major apoptosis protein, Bax can facilitate cell apoptosis by suppressing Bcl-2 activity. It is believed that a dynamic balance exists between Bax and Bcl-2. Bcl-2 as an oncogene-derived protein, confers negative control in the apoptosis pathway, while Bax, promotes cell death by competing with Bcl-2 (16). In this study, we showed that in rats pre-treated with spermine, Bax level increased 
significantly while Bcl-2 level decreased significantly. This suggested that spermine could protect nerve tissues in rats with ACIR injuries by inhibiting apoptosis.

We concluded that in rats with ACIR, spermine protected nerve tissues by decreasing the MDA level, increasing the activity of SOD, and modifying the balance between Bax and Bcl-2 proteins.

\section{References}

1. McBryde FD, Malpas SC and Paton JF: Intra-cranial mechanisms for preserving brain blood flow in health and disease. Acta Physiol (Oxf) 12: 46-47, 2016.

2. Liu H, Wei X, Kong L, Liu X, Cheng L, Yan S, Zhang X and Chen L: NOD2 is involved in the inflammatory response after cerebral ischemia-reperfusion injury and triggers NADPH oxidase 2-derived reactive oxygen species. Int J Biol Sci 11: 525-535, 2015.

3. Katayama Y, Inaba T, Nito C, Ueda M and Katsura K: Neuroprotective effects of erythromycin on cerebral ischemia reperfusion-injury and cell viability after oxygen-glucose deprivation in cultured neuronal cells. Brain Res 1588: 159-167, 2014.

4. Dong H, Wang S, Zhang Z, Yu A and Liu Z: The effect of mitochondrial calcium uniporter opener spermine on diazoxide against focal cerebral ischemia - reperfusion injury in rats. J Stroke Cerebrovasc Dis 23: 303-309, 2014.

5. Cui D, Wang L, Qi A, Zhou Q, Zhang X and Jiang W: Propofol prevents autophagic cell death following oxygen and glucose deprivation in PC12 cells and cerebral ischemia-reperfusion injury in rats. PLoS One 7: e35324, 2012.

6. Chen CM, Wu CT, Yang TH, Chang YA, Sheu ML and Liu SH: Green tea catechin prevents hypoxia/reperfusion-evoked oxidative stress-regulated autophagy-activated apoptosis and cell death in microglial cells. J Agric Food Chem 64: 4078-4085, 2016.
7. Wang T, Gu J, Wu PF, Wang F, Xiong Z, Yang YJ, Wu WN, Dong LD and Chen JG: Protection by tetrahydroxystilbene glucoside against cerebral ischemia: involvement of JNK, SIRT1, and NF-kappaB pathways and inhibition of intracellular ROS/RNS generation. Free Radic Biol Med 47: 229-240, 2009.

8. Ragnarsson L, Mortensen M, Dodd PR and Lewis RJ: Spermine modulation of the glutamate(NMDA) receptor is differentially responsive to conantokins in normal and Alzheimer's disease human cerebral cortex. J Neurochem 81: 765-779, 2002.

9. Yatin SM, Yatin M, Varadarajan S, Ain KB and Butterfield DA: Role of spermine in amyloid beta-peptide-associated free radical-induced neurotoxicity. J Neurosci Res 63: 395-401, 2001.

10. Zhang L, Gao X, Yuan X, Dong H, Zhang Z and Wang S: Mitochondrial calcium uniporter opener spermine attenuates the cerebral protection of diazoxide through apoptosis in rats. J Stroke Cerebrovasc Dis 23: 829-835, 2014.

11. He Y, Yang J, Li H, Shao H, Wei C, Wang Y, Li M and Xu C: Exogenous spermine ameliorates high glucose-induced cardiomyocytic apoptosis via decreasing reactive oxygen species accumulation through inhibiting $\mathrm{p} 38 / \mathrm{JNK}$ and JAK2 pathways. Int J Clin Exp Pathol 8: 15537-15549, 2015.

12. Lorente L, Martín MM, Pérez-Cejas A, Abreu-González P, Ramos L, Argueso M, Cáceres JJ, Solé-Violán J and Jiménez A: Association between total antioxidant capacity and mortality in ischemic stroke patients. Ann Intensive Care 6: 39, 2016.

13. Ye N, Liu S, Lin Y and Rao P: Protective effects of intraperitoneal injection of TAT-SOD against focal cerebral ischemia/reperfusion injury in rats. Life Sci 89: 868-874, 2011.

14. Wei H, Wei W, Bredesen DE and Perry DC: Bcl-2 protects against apoptosis in neuronal cell line caused by thapsigargin-induced depletion of intracellular calcium stores. J Neurochem 70: 2305-2314, 1998.

15. Zhao H, Yenari MA, Cheng D, Sapolsky RM and Steinberg GK: Bcl-2 overexpression protects against neuron loss within the ischemic margin following experimental stroke and inhibits cytochrome $\mathrm{c}$ translocation and caspase-3 activity. J Neurochem 85: 1026-1036, 2003

16. Yun Q, Jiang M, Wang J, Cao X, Liu X, Li S and Li B: Overexpression Bax interacting factor-1 protects cortical neurons against cerebral ischemia-reperfusion injury through regulation of ERK1/2 pathway. J Neurol Sci 357: 183-191, 2015. 